\title{
Finite Element Simulation of Frost Wedging in Ice Shelves
}

\author{
Carolin Plate $^{1, *}$, Ralf Müller ${ }^{1}$, Angelika Humbert $^{2}$, and Dietmar Gross ${ }^{3}$ \\ ${ }^{1}$ Institute of Applied Mechanics, University of Kaiserslautern \\ ${ }^{2}$ Section of Glaciology, Alfred-Wegener-Institut Helmholtz Zentrum für Polar- und Meeresforschung, Bremerhaven \\ ${ }^{3}$ Division of Solid Mechanics, TU Darmstadt
}

\begin{abstract}
Break-up events in ice shelves have been studied extensively during the last years. One popular assumption links disintegration events to surface melting of the ice shelf in conjunction with growing melt-water ponds, leading to hydro-fracture. As this explanation only holds during warm seasons [1], the possibility of frost wedging as forcing mechanism for autumn and winter break-up events is considered. Frost wedging can only occur if a closed ice lid seals the water inside the crack. Hence, the present study of frost wedging in a single crack uses ice lid thicknesses to evaluate the additional pressure on the crack faces. The investigation of the resulting stress intensity factor as a measure of crack criticality follows consequently. The results show that freezing water inside a crack can result in unstable crack growth of an initially stable water filled crack.
\end{abstract}

\section{Introduction}

(C) 2014 Wiley-VCH Verlag GmbH \& Co. KGaA, Weinheim

A sequence of satellite pictures acquired from July 2007 to April 2008 motivates the consideration of frost wedging as a possible break-up cause of huge ice shelf parts. Figure 1 shows a picture of the Wilkins Ice Shelf (WIS) with the area of interest, the ice shelf bridge, indicated in red. The marked area of the bridge in Fig. 2 remains intact until the 28th of February

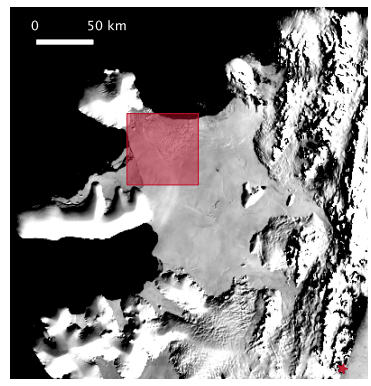

Fig. 1: WIS, MOA 2003/04

(C) NSIDC), star: FB station

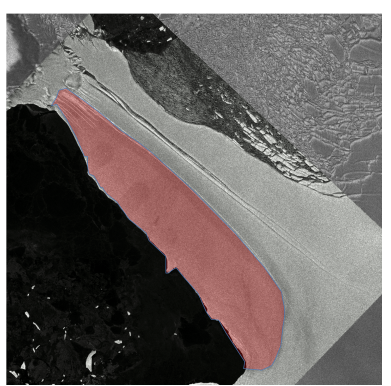

Fig. 2: Bridge, 30.07.2007, ALOS PALSAR, (c) TPMO)

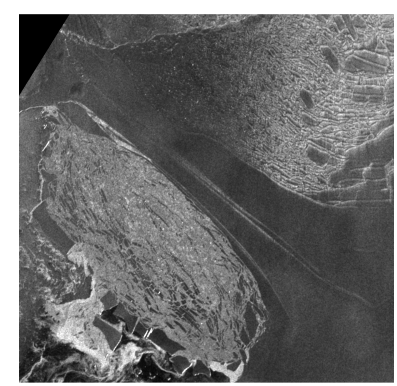

Fig. 3: Bridge, 07.03.2008, ENVISAT ASAR (c) ESA)

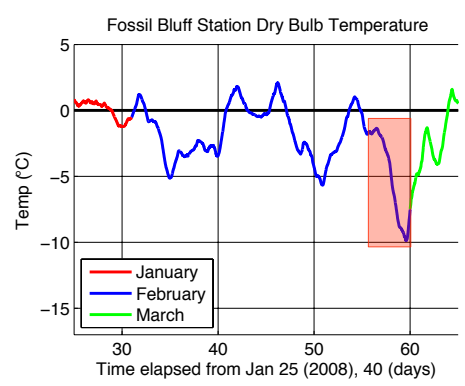

Fig. 4: Temperatures, Fossil Bluff (FB) Station, source: BAS

2008. Figure 3 shows the resulting bridge geometry after the 29th of February 2008 and the floating ice shelf fragments. A plot of the surface temperatures during the break-up period from a research station close to the Wilkins Ice Shelf is shown in Fig. 4. The break-up event coincides with the pronounced temperature drop, indicated in red, at the end of February. Different measurements show, that the weather (especially the wind speed) was moderate during that time.

\section{Modeling Approach}

The geometric dimensions of an ice shelf (100 m-500 m thickness, several hundreds of kilometers in width) allow a plane strain simulation of an infinite crack in a cross section of an infinite ice shelf as illustrated in Fig. 5 . The fractured $2000 \mathrm{~m}$

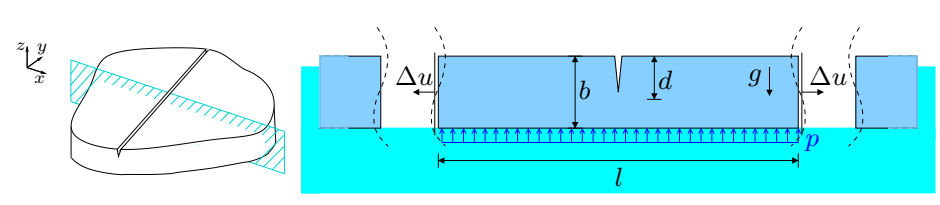

Fig. 5: Plane strain model geometry with applied boundary conditions
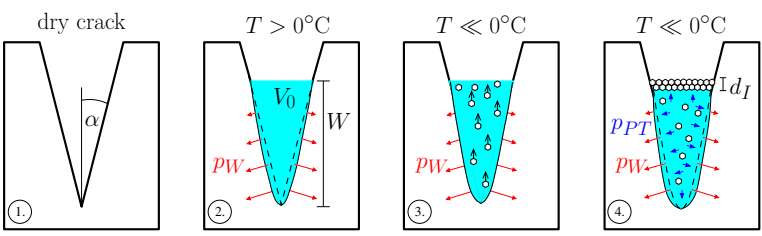

Fig. 6: Frost wedging mechanism

long and $250 \mathrm{~m}$ thick part of the ice shelf is loaded by gravity and by a water and phase transition pressure on the crack faces. The boundary condition at the bottom ensures floating and a displacement boundary condition at the vertical boundaries allows for a loading equivalent to a measured viscous surface stress $\sigma_{v}=100 \mathrm{kPa}$. Measurements of the fracture behavior of ice shelf ice indicate a linear elastic material answer for the short time span during which the fracture process occurs. The elastic constants used in the simulations are Young's modulus $E=9 \mathrm{GPa}$, and Poisson's ratio $\nu=0.3$. A depth dependent density

\footnotetext{
* Corresponding author: e-mail plate@ rhrk.uni-kl.de
} 
profile is applied. The crevasse is modeled as a notch with an opening angle $\alpha$ ending in a sharp Griffith crack tip to ensure the applicability of established fracture mechanical concepts. For more details on the fracture mechanical analysis of cracks in ice shelves, the reader is referred to [2]. Figure 6 illustrates the simulated frost wedging mechanism where a stable dry crack of length $d=65 \mathrm{~m}$ is filled with melt-water up to a water level $W$. The crack is still stable. The volume per thickness increment of water inside the crack in the deformed configuration, $V_{0}$ is computed. Now, we assume that freezing begins, ice crystals form in the water and rise to the surface where they eventually build a closed lid of the thickness $d_{I}$. The displaced water under the lid enforces the aggregation of an additional pressure on the crack faces and the lid, $p_{P T}$. This pressure can be computed using a Newton's iteration solving the equation $V\left(p_{P T}\right)=V_{0}-V_{I} \frac{\rho_{I}}{\rho_{W}}$, where $V_{I}$ is the volume per thickness increment of the ice lid, $\rho_{I}$ and $\rho_{W}$ are the densities of ice and water, respectively, and $V\left(p_{P T}\right)$ is the remaining water volume per thickness increment under the lid. Once the appropriate pressure $p_{P T}$ is evaluated, configurational forces can be used to compute the stress intensity factors $K_{I}$ at the crack tip. For $K_{I}>K_{I c}=4 \mathrm{kPa} \sqrt{\mathrm{m}}$ the Griffith crack tip grows until $K_{I}<K_{I c}$ or $d=250 \mathrm{~m}$. If a new stable crack configuration is reached, the ice-lid thickness is increased again.

\section{Results}

Figures 7 to 10 show the additional pressure $p_{P T}$, the stress intensity factors $K_{I}$ and the crack depth $d$ resulting from given ice thickness increments $d_{I}$ plotted over the simulation steps (crack growth or ice-lid growth) exemplary for four different setups. The plots differ in the water level and the opening angle. Figure 7 shows, that freezing water in an ideally sharp crack $\left(\alpha=0^{\circ}\right)$ will only lead to little crack growth until the crack is fully frozen. Cracks with an opening angle $\alpha>0^{\circ}$ show a very different behavior. For $\alpha=1 / 10^{\circ}$ and $W=10 \mathrm{~m}$ as shown in Fig 8 an ice lid with $d_{I}=0.6 \mathrm{~m}$ is sufficient to allow the crack to break through, once a critical water column is reached, even without additional pressure. The results for larger opening angles are more difficult to interpret, as here the stress intensity factors at the crack tip as well as the tensile stresses in the lid are very high. A prediction which part collapses first needs further information of the bridge geometry and the freezing mechanism. Hence, only results for geometries with sub critical bridge stresses are presented. Figures 9 and 10 show the results for a $0.1^{\circ}$ and a $0.2^{\circ}$ opening angle with $W=5 \mathrm{~m}$ water column, respectively. With the $0.1^{\circ}$ opening, the crack freezes completely, while the $0.2^{\circ}$ open crack breaks completely with $1.8 \mathrm{~m}$ of ice lid thickness.

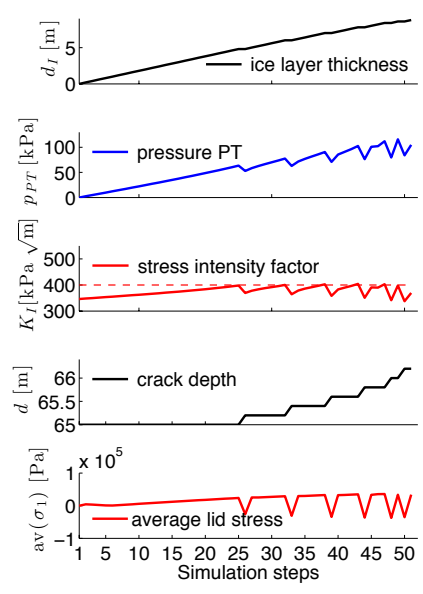

Fig. 7: $\alpha=0, W=10$
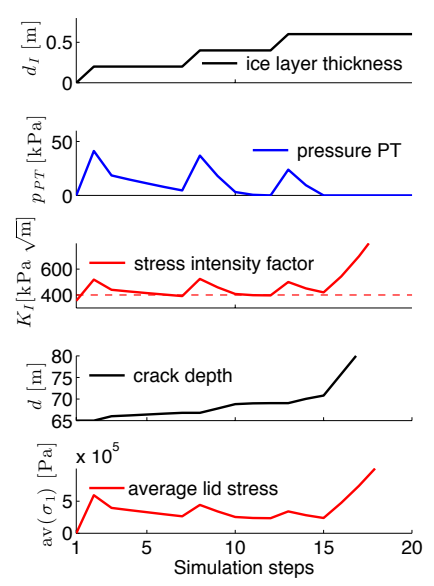

Fig. 8: $\alpha=1 / 10^{\circ}, W=10$

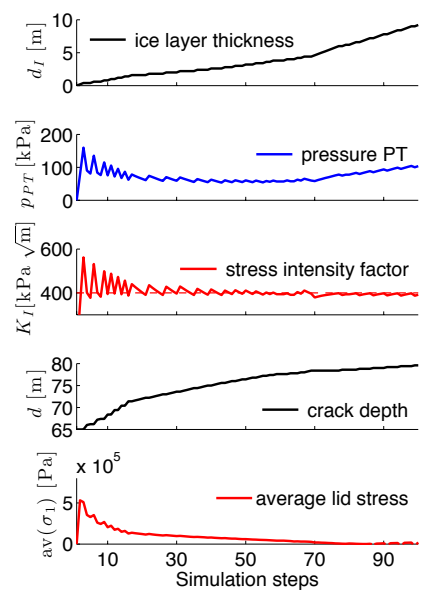

Fig. 9: $\alpha=1 / 10^{\circ}, W=5$
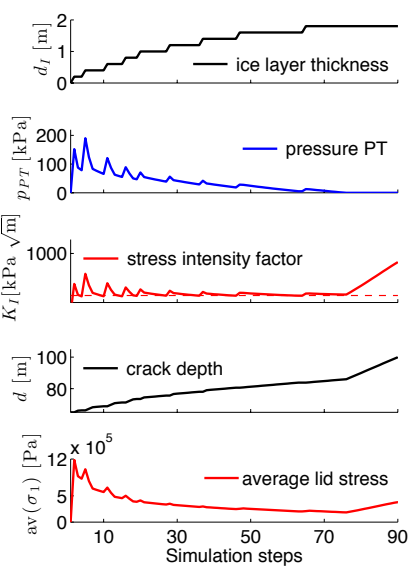

Fig. 10: $\alpha=2 / 10^{\circ}, W=5$

\section{Conclusion}

The simulations show that frost wedging can be considered as a triggering mechanism for autumn an winter disintegration events. However, the pronounced influence of the opening angle and the required sealed crevasses reveal the need for more informations about crevasse geometries and the freezing process inside a crevasse. Further investigations should consider freezing at the crack tip and a three-dimensional extension of the model.

Acknowledgements This work was supported by the DFG priority program Antarktisforschung, SPP1158, project number MU1370/4-1.

\section{References}

[1] M. Braun and A. Humbert, Recent Retreat of Wilkins Ice Shelf Reveals New Insights in Ice Shelf Breakup Mechanisms, IEEE Geoscience and Remote Sensing Letters 6, 263-267 (2009).

[2] C. Plate, R. Müller, A. Humbert and D. Gross, Evaluation of the criticality of cracks in ice shelves using finite element simulations, The Cryosphere 6, 973-984 (2012). 